Erratum

\title{
High Expression of Cancer-Derived Glycosylated Immunoglobulin G Predicts Poor Prognosis in Pancreatic Ductal Adenocarcinoma: Erratum
}

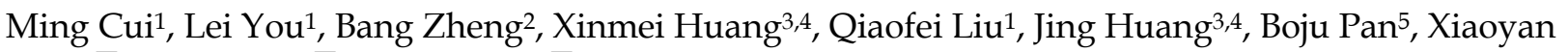
Qiu $^{3,4}{ }^{\bowtie}$, Quan Liao $^{1 凶}$, Yupei Zhao ${ }^{\circledR}$

1. Department of General Surgery, Peking Union Medical College Hospital, Chinese Academy of Medical Sciences \& Peking Union Medical College, Beijing 100730, China

2. School of Public Health, Faculty of Medicine, Imperial College London, London W6 8RP, UK

3. Department of Immunology, School of Basic Medical Sciences, Peking University, Beijing 100191, China

4. Peking University Center for Human Disease Genomics, Beijing 100191, China

5. Department of Pathology, Peking Union Medical College Hospital, Chinese Academy of Medical Sciences \& Peking Union Medical College, Beijing 100730, China

$\triangle$ Corresponding authors: Quan Liao and Yu-Pei Zhao, Peking Union Medical College Hospital, Beijing 100730, China. E-mails: lqpumc@126.com, zhao8028@263.net and Xiao-Yan Qiu, Peking University, Beijing 100191, China. Email: qiuxy@bjmu.edu.cn

(c) The author(s). This is an open access article distributed under the terms of the Creative Commons Attribution License (https://creativecommons.org/licenses/by/4.0/). See http://ivyspring.com/terms for full terms and conditions.

Published: 2021.09 .05

Corrected article: J Cancer 2020; 11 (8): 2213-2221. doi: 10.7150/jca.39800.

In the original version of the article, the representative image of siRNA2-silenced T3M4 cells in Figure 3G is incorrect. The correct Figure 3G is as follows. This correction will not affect the results and conclusions. The authors would like to apologize for any inconvenience this may have caused.
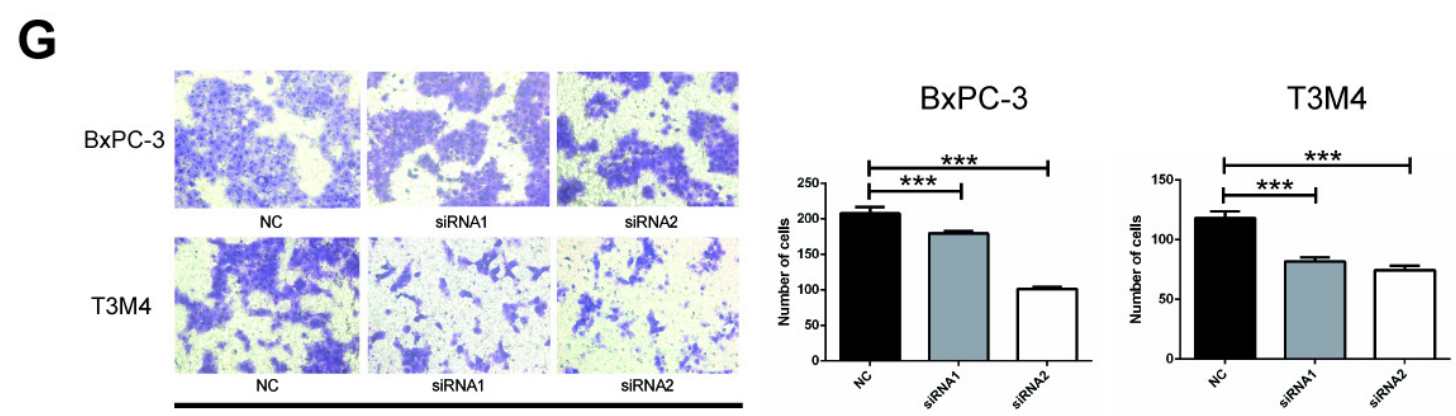

Invasion

Figure 3. (G) BxPC-3 and T3M4 cells treated with siRNA were subjected to invasion assay. Representative images are shown for each group. Original magnification, 200x. $* * P<0.01, * * * P<0.001$

\section{References}

1. Cui M, You L, Zheng B, et al. High Expression of Cancer-Derived Glycosylated Immunoglobulin G Predicts Poor Prognosis in Pancreatic Ductal Adenocarcinoma. J Cancer. 2020;11(8):2213-2221. 